\title{
Corner Sort for Pareto-based Many-Objective Optimization
}

\author{
Handing Wang, Student Member, IEEE, Xin Yao, Fellow, IEEE,
}

\begin{abstract}
Non-dominated sorting plays an important role in Pareto-based multi-objective evolutionary algorithms (MOEAs). When faced with many-objective optimization problems (multiobjective optimization problems (MOPs) with more than 3 objectives), the number of comparisons needed in non-dominated sorting becomes very large. In view of this, a new corner sort is proposed in this paper. Corner sort first adopts a fast and simple method to obtain a non-dominated solution from the corner solutions, and then uses the non-dominated solution to ignore the solutions dominated by it to save comparisons. Obtaining the nondominated solutions requires much fewer objective comparisons in corner sort. In order to evaluate its performance, several stateof-the-art non-dominated sorts are compared with our corner sort on three kinds of artificial solution sets of MOPs and the solution sets generated from MOEAs on benchmark problems. On the one hand, the experiments on artificial solution sets show the performance on the solution sets with different distribution. On the other hand, the experiments on the solution sets generated from MOEAs show the influence different sorts bring to MOEAs. The results show that corner sort performs well especially on many-objective optimization problems. Corner sort uses fewer comparisons than others.
\end{abstract}

Index Terms-Non-dominated sort, Pareto based MOEA, many-objective optimization, corner sort

\section{INTRODUCTION}

Non-dominated sorting is an essential component of Paretobased MOEAs, which plays an important role in the development of Pareto-based MOEAs. Pareto-based MOEA is a kind of MOEAs with the selection operator based on Pareto dominance, such as MOGA [1], NSGA[2], NSGA-II [3], NPGA [4], SPEA [5], SPEA2 [6], PAES [7], PESA-II [8], MOPSO [9], and TDEA [10]. As the development of Paretobased MOEAs so far, non-dominated sorting is still a key topic of Pareto-based MOEAs, because most computation cost of Pareto-based MOEAs comes from non-dominated sorting.

Non-dominated sorting is a process of assigning solutions to different ranks. According to Pareto dominance, the solutions in the same rank are non-dominated by each other and they are dominated by at least one solution in their former rank. Non-dominated sorting is required to output the right Pareto dominance rank. All the non-dominated sorts output the same result by inputting the same solution set. The only difference is their different computational cost. The time complexity of the native non-dominated sorting is $O\left(m N^{3}\right)$, where $m$ is the number of objectives and $N$ is the magnitude of solution set. In 2002, Deb [3] proposed a fast non-dominated sort, which lowers the complexity to $O\left(m N^{2}\right)$. However, there is still some waste on some unnecessary comparisons.

Many-objective optimization problem is a kind of special MOPs with more than 3 objectives [11]. Its large number of objectives increase its difficulties for Pareto-based MOEAs due to their weak Pareto dominance selection pressure [12], [13], [14], [15]. Objective reduction [16], [17], and scalarization methods [18] are some strategies to reduce the difficulties of the original problems. Also, many MOEAs aim to solve many-objective optimization problems, such as IBEA [19], HypE [20], and MODELS [21]. Among the studies of manyobjective optimization, dominance strategy and ranking are mainly focused on. For example, a controlling dominance area is proposed to strengthen the selection for many-objective optimization problems in [22]; a epsilon-ranking is adopted to improve Pareto dominance in [23]; a kind of fuzzy dominance is applied in [24], [25]; different kinds of data structure and representation increase the speed of ranking in [26], [27].

In view of the characteristics of many-objective optimization problems, non-dominated sort has to face new challenges because of the large number of objective comparisons. The challenges come from two aspects. Firstly, as the number of objectives increases, more objective comparisons are needed to determine their dominance relation. Secondly, the existing comparison saving method cannot work effectively on manyobjective optimization problems, because there are fewer dominated solutions to be ignored in the solution set. In view of this, a corner sort is proposed specially for the comparison saving of many-objective optimization problems. Thus, MOEAs can save more computational cost (both time and space) for solving many-objective optimization problems. The common comparison saving method is also adopted in corner sort. Corner sort saves the comparisons in the process of obtaining the non-dominated solutions. It discards the solutions dominated by the non-dominated solutions in the sorting for the current rank. The contributions of this paper include the following.

- Corner sort adopts a simple and fast way to obtain non-dominated solutions according to a characteristic of MOPs (the solution of the best objective is nondominated). It only requires $N-1$ objective comparisons for the set of $N$ solutions. The corner solutions are preferentially selected to obtain the non-dominated solutions, which is the reason why the proposed sort is called "corner sort".

- In our experiments, the number of the comparisons between two objectives rather than the number of the dominance comparisons between two solutions is employed to evaluate the performance of the different non-dominated sorts, which describes the computational cost fairly.

The rest of this paper is organized as follows. The related 


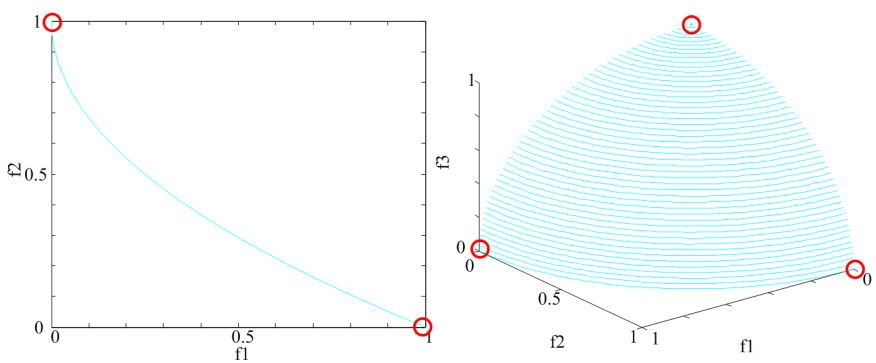

Fig. 1. Examples of corner solutions.

concepts will be introduced in section II. The basic idea and details of corner sort are given in section III. Section IV is the experimental part to show the performance of corner sort. Finally, section V concludes the whole paper.

\section{BACKGROUND}

\section{A. Pareto Dominance}

Pareto dominance is defined for comparing two solutions with multiple objectives, which is the base of non-dominated sorting. One vector is said to dominate another one only if all of its objectives are not worse than another one's. Vectors $x=\left(x_{1}, \ldots, x_{i}, \ldots, x_{m}\right)$ and $y=\left(y_{1}, \ldots, y_{i}, \ldots, y_{m}\right)$ are two solutions of a minimization problem with $m$ objectives. If $x_{i} \leq$ $y_{i}(1 \leq i \leq m)$ and $x \neq y, x$ dominates $y$, written as $x \prec y$.

Usually, dominance comparison is applied by comparing the objective values of two solutions sequentially. Theoretically, it needs $m$ objective comparisons to determine the relation between two solutions for an MOP with $m$ objectives. In fact, an effective method is applied in all the existing nondominated sort. As the objective comparisons are serial, the dominance relation is regarded as "non-dominated" once meeting conflict. Thus, it needs 2 to $m$ objective comparisons. In other words, the cost of different solutions is different. It is unfair to employ the number of comparisons of two solutions to evaluate the performance of non-dominated sort. In the experiment of section IV, we employ the number of objective comparisons to evaluate the performance of different non-dominated sorts.

\section{B. Corner Solutions}

For an MOP with $m$ objectives, the solution which is considered $k$ objectives $(k<m)$ in the $m$-objective space is called a corner solution [28]. In this paper, we only consider the corner solutions with $k=1$. There are some examples of corner solutions in Figure 1.

\section{Existing Non-Dominated Sort Algorithms}

Non-dominated sort is one of the most complicated processes in Pareto-based MOEAs. Its natural time complexity is $O\left(m N^{3}\right)$, which is too high for the solution set of a large scale. All non-dominated sorts aim at obtaining the right dominance rank of the solution set at the same time of lowering the number of comparisons.
Fast non-dominated sort [3] is the first fast algorithm to lower the complexity to $O\left(m N^{2}\right)$. As [29] shows, No-FreeLunch does not hold all the time. We can still find generally outperforming sorting algorithms. There are still some unnecessary comparisons in fast non-dominated sort. In the later study, researchers devoted themselves to reducing the unnecessary comparisons in the sorting. Among their research, non-dominated rank sort [30] and deductive sort [31] are the examples of $O\left(m N^{2}\right)$ non-dominated sorts with some comparison saving strategies.

1) Fast Non-Dominated Sort : Fast non-dominated sort [3] is the promotion of the development of Pareto-based MOEAs. It first travels all of the solutions to obtain all the dominance relation between every two solutions. According to such relation, the dominance rank is assigned finally. Fast non-dominated sort need at most $m N(N-1)$ objective comparisons For every solution $p$ in fast non-dominated sort, list $S_{p}$ stores the solutions which are dominated by solution $p$, and counter $n_{p}$ records the number of the solutions which dominate solution $p$. The space complexity is $O\left(N^{2}\right)$. When the scale of solution set increases, both time and space complexity increase rapidly.

2) Non-Dominated Rank Sort : Non-dominated rank sort in [30] is a sort with comparison saving strategy, which is faster than fast non-dominated sort. It sorts the non-dominated solutions in the current solution set sequentially. If one solution is dominated by any solution in the current solution set, its following comparisons to the rest solutions are ignored. If one solution is non-dominated in the current solution set, it is marked as the current rank and deleted in the current solution set. In the worst case, non-dominated rank sort requires $m N(N-1) / 2$ objective comparisons. The time complexity of non-dominated rank sort is still $O\left(m N^{2}\right)$. It does ignore some unnecessary comparisons for the dominated solutions, which requires less cost than fast non-dominated sort. Furthermore, its space complexity is only $O(N)$.

3) Deductive Sort : Although non-dominated rank sort requires fewer comparisons, it still wastes some comparisons. For example, if $a \prec b, b \prec c$, then $a \prec c$. The comparisons between $a$ and $c$ is unnecessary. Deductive sort [31] aims at saving these comparisons. On the one hand, it ignores the comparisons of the dominated solutions to the rest solutions as non-dominated rank sort. On the other hand, it marked and ignored any dominated solutions in the ranking of current rank. Similar to non-dominated rank sort, deductive sort requires $m N(N-1) / 2$ objective comparisons in the worst case. Its time complexity is $O\left(m N^{2}\right)$ and its space complexity is $O(N)$.

\section{CORNER SORT}

\section{A. Basic Idea}

The basic idea of saving comparisons in corner sort is to use the non-dominated solutions to ignore the solutions which they dominate. Two processes of corner sort combine together for the aim of comparison saving. One is ignoring the dominated solutions as that in deductive sort. The other one is obtaining the non-dominated solutions, which is unique to 
corner sort. Naturally, it needs $m(N-1)$ at most and $2(N-1)$ at least objective comparisons for obtaining a non-dominated solution in the solution set of $N$ solutions. We wonder if some characteristics of MOP can help to save comparisons in this process. We find that the solutions with the best objective are definitely non-dominated. In other words, corner solutions [28] are selected preferentially for non-dominated solutions.

Because only $N-1$ comparisons on only one objective are needed for obtaining a non-dominated solution, it is much fewer than the $m(N-1)$ objective comparisons especially for many-objective optimization problems. Although it saves comparisons, its time complexity is still $O\left(m N^{2}\right)$. Its space complexity is $O(N)$.

After obtaining one non-dominated solution, corner sort marks the solutions dominated by the obtained non-dominated solution to ignore in the later sort of the current rank. As the non-dominated solutions have been ranked, their comparisons to the unmarked solutions are only to check whether they dominates the unmarked solutions.

\section{B. Corner Sort}

The implementation of corner sort is a loop of two steps, obtaining a non-dominated solution and marking the solutions which are dominated by the non-dominated solution. For an MOP with $m$ objectives, at least $m$ non-dominated solutions are available. For a sequential process, only one non-dominated solution is needed for each time. It is worth discussing the order of choosing non-dominated solutions. The best order depends on the distribution of the solution set. However, the situation cannot be estimated in advance. The uniform distribution is the average of all the situations. Therefore, we use the solution set with a kind of uniform distribution for all the situations to analyze this problem. There are two cases as shown in Figure 2, where the white points are non-dominated solutions and the light grey area is the area that they dominate. Case A is to choose the non-dominated solutions in a looping order and Case B is to choose the non-dominated solutions always in one objective. In Figure 2 , it is clear that the marked area in Case A is larger than that of Case B. The looping order is adopted for choosing the non-dominated solutions in corner sort. It does not mean Case B is useless. In some particular situations that there are more solutions around objective $f_{1}$, Case B performs better than Case A. However, if there are more solutions around other objectives, Case B performs least-effectively. Taken all these different kinds of distribution into consideration, it is impossible to require that our sort has the best efficiency in all different situations. Case A is not the best order for all the situations but the most robust order, because it never acts too badly on these solution sets with any particular distribution.

The pseudo code of corner sort is given in Table I. In Table I, if the non-dominated solution is obtained from objective $f_{j}$, objective $f_{j}$ is ignored in its later comparisons to other unmarked solutions. As the non-dominated solution has the best objective $f_{j}$, the comparisons on objective $f_{j}$ is unnecessary. In order to explain the process of corner sort, an sorting example is included in Figure 3. Firstly, all the solutions in $P$ are unmarked, corner sort obtains the nondominated solution and marks the solutions that the nondominated solution dominates. It chooses the non-dominated solutions in a looping order as in subfigure A. It finishes sorting of rank one when all the solutions are all marked as in subfigure B, the white points are in rank one. After that all the unranked solutions are unmarked for the sorting of the next rank as in subfigure C. Corner sort loopingly acts as above until all the solutions are ranked.

\section{Corner Sort for Many-Objective Optimization Problems}

For many-objective optimization problems, the cost of nondominated sorting increases rapidly with the number of objectives. The comparisons between two solutions of manyobjective optimization problems are very expensive, because they have more objectives to be compared. In many-objective optimization problems, the solutions are hard to be dominated. Thus, there is less chance to save comparisons by ignoring the dominated solutions. Corner sort aims to save comparisons from the process of obtaining the non-dominated solutions. It only requires $N-1$ comparisons on one objective. Normally, 2 to $m$ objective comparisons are needed for the comparison of two solutions. $N-1$ comparisons on one single objective are definitely fewer than $m(N-1)$ objective comparisons. The more objectives one MOP has, the more objective comparisons corner sort can save.

In the real world, there are many-objective optimization problems based on preference. Corner sort is suitable for the preference based many-objective optimization. The order of choosing non-dominated solution can be referred to preference information (such as weight vector and lexicographic minimum). In this way, the sorting is more efficient.

\section{EXPERIMENTAL STUdiES}

We test the performance of different non-dominated sorts on both artificial datasets and the solution sets from MOEAs. On the one hand, the artificial datasets describe the situation that MOEAs may meet. As only the objective values are considered in non-dominated sorts, the artificial datasets only include objective values. The experiments on those datasets aim to analyze the behaviors of different sorts on the solution sets with particular distribution. On the other hand, the experiments on the solution sets from MOEAs aim to show the influence that different sorts bring to MOEAs. Fast non-dominated sort [3], non-dominated rank sort [30], and deductive sort [31] are employed as the compared algorithms in our experiment. As the process of obtaining non-dominated solutions does not need to compare all the objectives of two solutions, it is not available to adopt the number of comparisons between two solutions to evaluate the performance. We use the number of objective comparisons and execution time to evaluate the performance of different non-dominated sorts. It is worth noting that the objective comparisons for obtaining non-dominated solutions in corner sort are included in the total number of objective comparisons. Since all the non-dominated sorts obtain the same Pareto dominance rank for the same solution set and the aim of our experiments is to evaluate the efficiency 
A: Loop in all the objectives

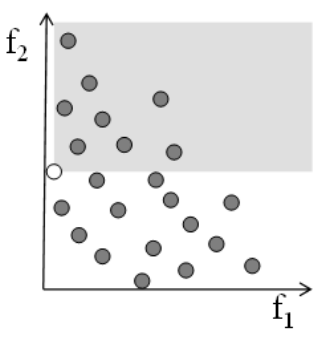

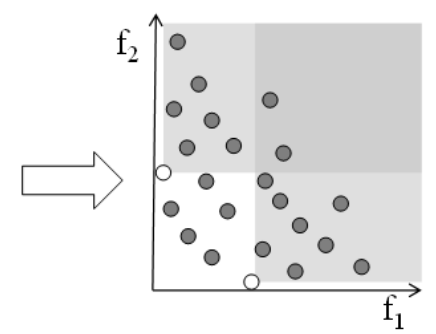

B: Always in one objective

Fig. 2. Two cases of choosing non-dominated solution.
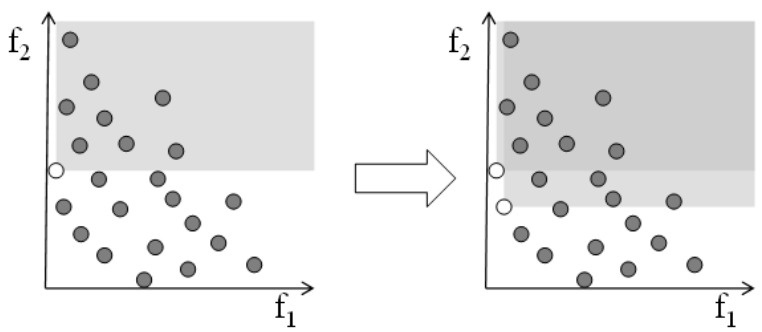

TABLE I

PSEUDO CODE OF CORNER SORT.
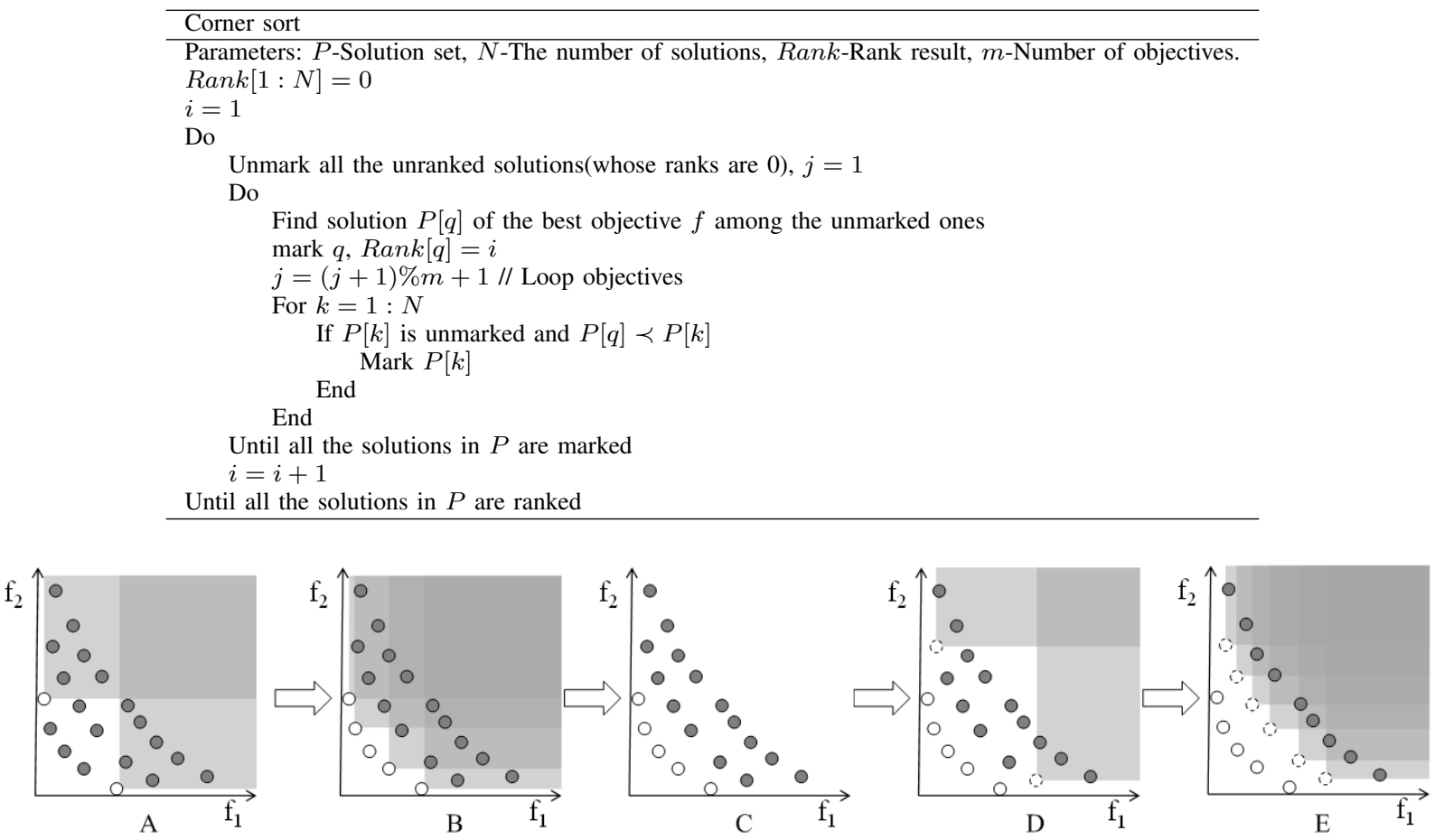

Fig. 3. An example of corner sort. Subfigure A: Corner solutions are ranked and the solutions dominated by them are ignored. Subfigure B: Ranking for the first rank is finished. Subfigure C: The unranked solutions are unmarked. Subfigure D: Corner solutions of the rest unmarked solutions are ranked and the solutions dominated by them are ignored. Subfigure E: Ranking for the second rank is finished.

of different sorts, the results of ranking are not included in this paper.

\section{A. Experiments on Cloud Dataset}

1) Cloud Dataset: Cloud dataset is a dataset of uniformrandom distributed solutions, which describes the random situation in Pareto-based MOEAs. The sample creation algorithm is shown in Table II. Such a case usually occurs at the beginning of MOEAs. The relation between the number of fronts and the number of objectives is shown in Figure 4. With the increasing of objectives, the number of fronts decreases rapidly, because most solutions are non-dominated by each other in many-objective optimization problems.
TABLE II

SAMPLE CREATION ALGORITHM FOR CLOUD DATASET.

\begin{tabular}{l} 
Sample creation algorithm for cloud dataset \\
\hline Parameters: $N$-The number of solutions, $m$-The number of objectives, \\
$\mathrm{U}(0,1)$-Uniformly distributed number in $[0,1]$ \\
For $i=1: N$ \\
For $j=1: m$ \\
$\quad$ Dataset $(i, j)=\mathrm{U}(0,1)$ \\
End \\
End
\end{tabular}

2) Results: The computational complexity of nondominated sort comes from two aspects, numbers of solutions and objectives. The results are divided into two parts, one is the experiment on the dataset of a fixed number of objectives 


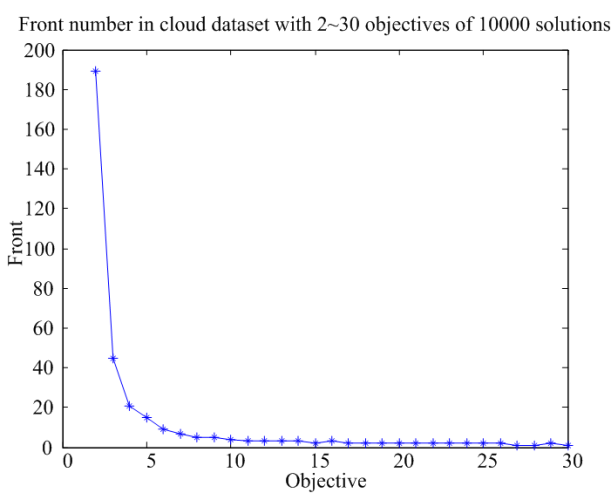

Fig. 4. The number of fronts in the cloud dataset with 2-30 objectives of 10000 solutions.

with variable numbers of solutions and the other one is the experiment on the dataset of a fixed number of solutions with variable numbers of objectives.

Figure 5 is the result of numbers of objective comparisons with variable numbers of solutions. According to Figure 5, fast non-dominated sort requires the most objective comparisons. Non-dominated rank sort requires fewer objective comparisons than fast non-dominated sort but more than deductive sort and corner sort. For low-dimensional objectives such as two objectives, deductive sort requires fewer comparisons than corner sort. For high-dimensional objectives, deductive sort requires more comparisons than corner sort. Also, the performance of deductive sort and corner sort on the cloud dataset of different numbers of objectives are different by Figure 5 . This is because the dominance relation in the cloud dataset with different numbers of objectives is different. Since there are more fronts in the 2-objective cloud dataset, deductive sort can ignore more dominated solutions. Because of its ignoring strategy, deductive sort ignores solutions in a random way. Deductive sort requires fewer objective comparisons than corner sort in the low-dimensional cases.

Figure 6 shows the average execution time of 4 nondominated sorts on cloud dataset with different numbers of solutions from 30 independent runs. Fast non-dominated sort costs the most execution time, which is the same as the result of numbers of comparisons. Deductive sort costs more time than non-dominated rank sort. Corner sort costs the least time in most cases.

According to Figures 5 and 6, all the sorts require more computational cost with the increasing numbers of solutions. Fast non-dominated sort increases most rapidly. Nondominated rank sort increases more slowly than fast nondominated sort, because it discards the non-dominated ones in the later sorting. As both deductive sort and corner sort ignore the dominated solutions in the sorting of one rank, they use fewer objective comparisons.

The left subfigure in Figure 7 shows the result of numbers of objective comparisons with variable numbers of objectives. The number of objective comparisons of all the non-dominated sorts increase with the objective number. For the datasets with more than 15 objectives, the objective comparison stops increasing, because cloud datasets with more than 15 objectives
TABLE III

SAMPLE CREATION ALGORITHM FOR FIXED FRONT DATASET.

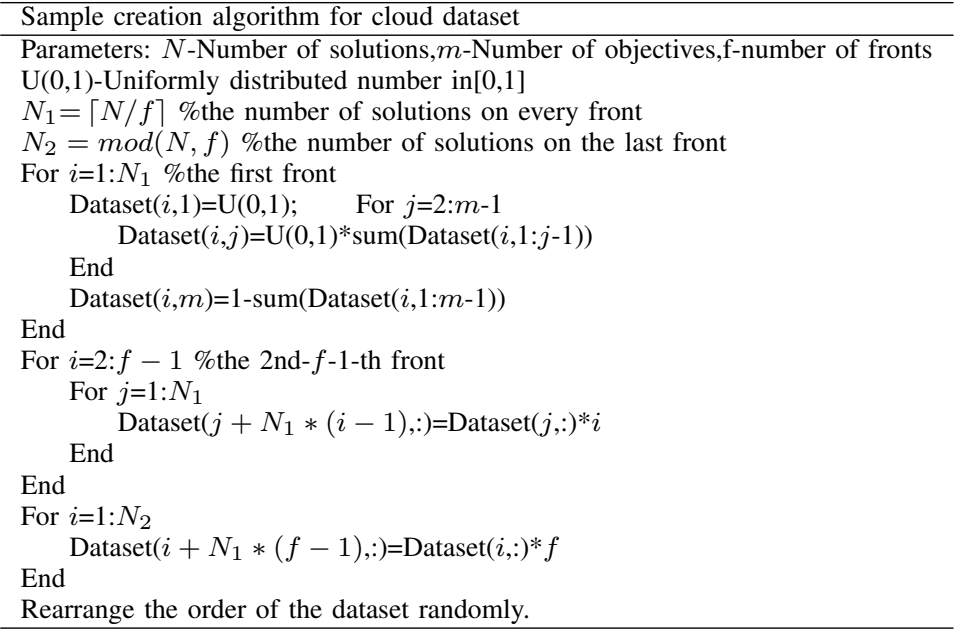

have only one front, which means there are no dominated solutions to be ignored. It is worth noting that the number of objective comparison of deductive sort increases faster than that of corner sort. Though corner sort requires more comparisons than deductive sort for MOPs with low-dimensional objectives, it requires fewer comparisons for MOPs with highdimensional objectives. The right subfigure in Figure 7 shows the average execution time with variable numbers of objectives from 30 independent runs. It costs a little bit more execution time for the dataset with more objectives. Fast non-dominated sort costs the most execution time, and corner sort costs the least execution time.

\section{B. Experiments on Fixed Front Dataset}

1) Fixed Front Dataset: Fixed front dataset [31] is a kind of dataset with a controllable number of fronts, which describes the situation in Pareto-based MOEAs when the search mainly focuses on fronts. In the fixed front dataset, solutions are divided into a fixed number of fronts with almost the same size. Every front is distributed on a line or a plane as in Figure 8 . The details of the sample creation algorithm are shown in Table III. In order to understand this dataset deeply, we take an example with $F$ fronts and $n$ solutions. For the solution in rank $R$, there are $n(F-R) / F$ solutions which are dominated by that solution. The solutions are distributed in different fronts with a same probability. Thus, the expectation of the solutions can be dominated is $\sum_{R=1}^{F} \frac{n}{F} \frac{F-R}{F}=n\left(\frac{1}{2}-\frac{1}{2 F}\right)$, which is shown in Figure 9.

2) Results: In the experiment on the fixed front dataset, two factors influence the behavior of non-dominated sort very much. One is the number of objectives, the other is the number of fronts. The following experiment has two parts for those two factors. As the influence of the number of solutions is analyzed in the experiment of cloud dataset. The fixed front datasets employed here are of a fixed number of solutions (7500).

Figure 10 is the result of numbers of comparisons on the fixed front dataset with variable numbers of fronts. The num- 

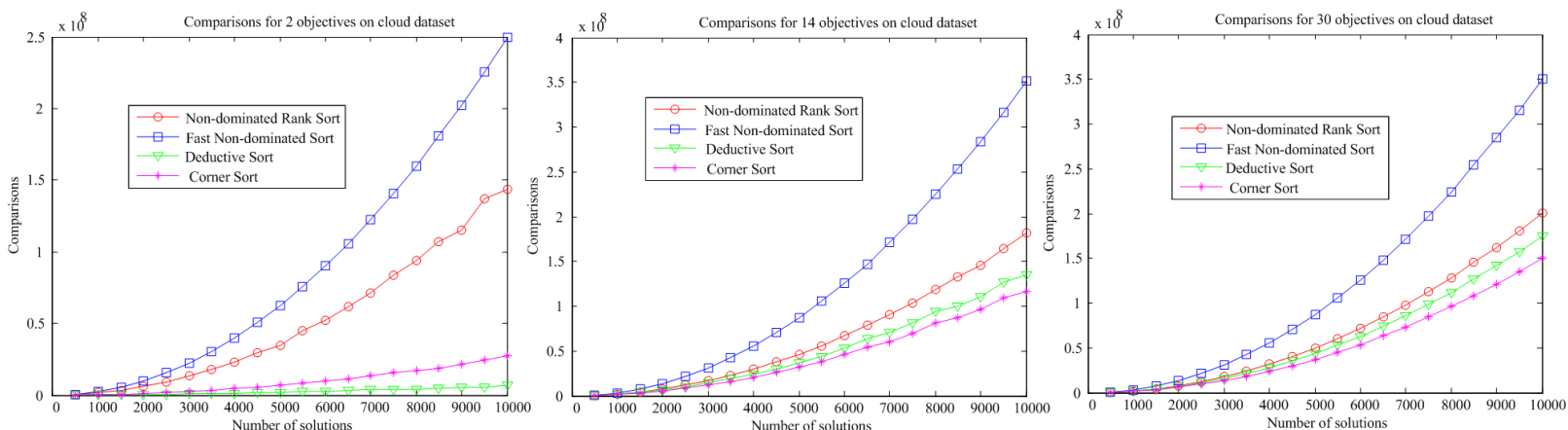

Fig. 5. Comparisons of 4 non-dominated sort on 2, 14, 30 objective cloud datasets with different numbers of solutions.
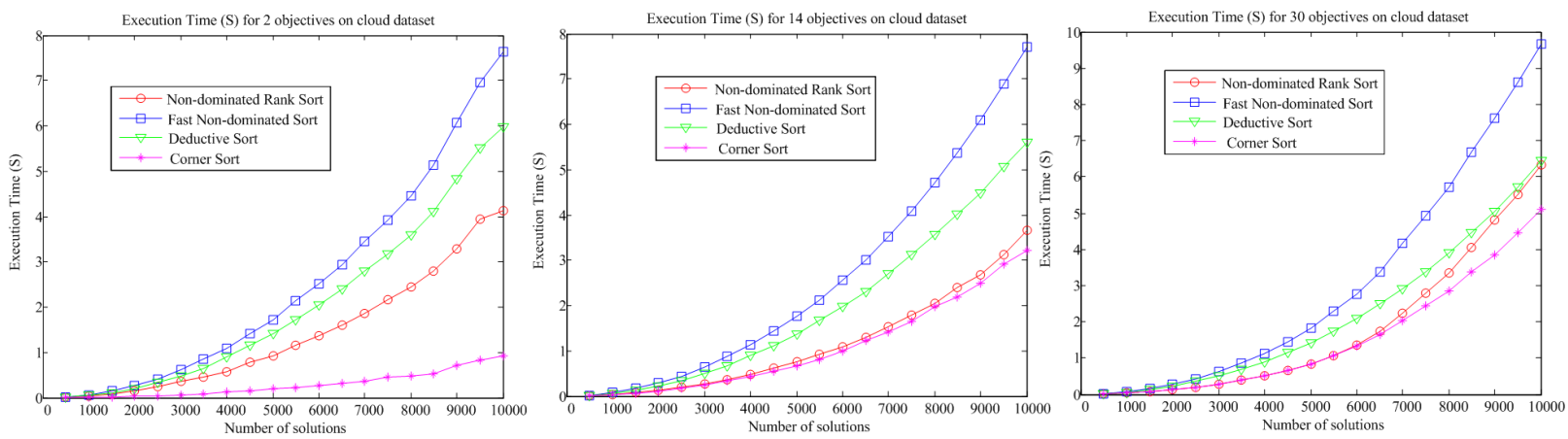

Fig. 6. Average execution time of 4 non-dominated sort on 2, 14, 30 objective cloud datasets with different numbers of solutions from 30 independent runs.
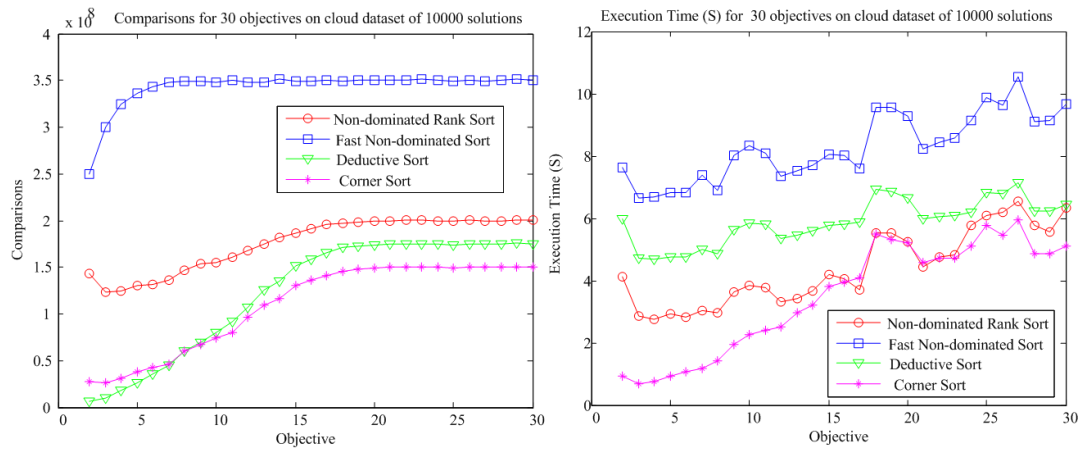

Fig. 7. Results of 4 non-dominated sort on 2-30 objective cloud datasets of 10000 solutions.

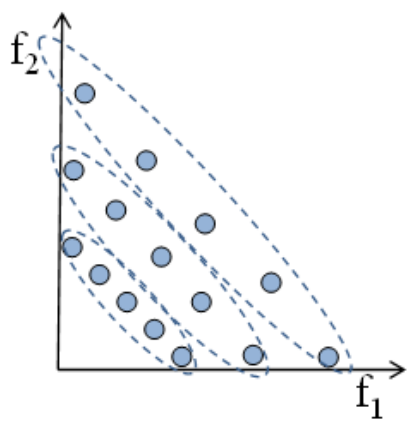

Fig. 8. An example of fixed front dataset is with 3 fronts and 15 solutions. The solutions in the area of dotted lines are in the same front. bers of comparisons of all the sorts drop with the increasing number of fronts except for fast non-dominated sort, because there are more solutions which can be dominated in the datasets with larger number of fronts as Figure 9. It is clear that fast non-dominated sort requires the most comparisons in all cases of the experiment. Deductive sort and corner sort require fewer comparisons than non-dominated rank sort. In most cases, corner sort requires fewer comparisons than deductive sort except for the cases with low-dimensional objectives. It needs 2 to $m$ objective comparisons between two solutions. For the high-dimensional cases in Figure 10, we find that the numbers of objective comparison of fast non-dominated sort and non-dominated rank sort increase a little bit with the number of fronts after their decreasing, because they do not ignore the dominated solutions. Fast non-dominated sort 


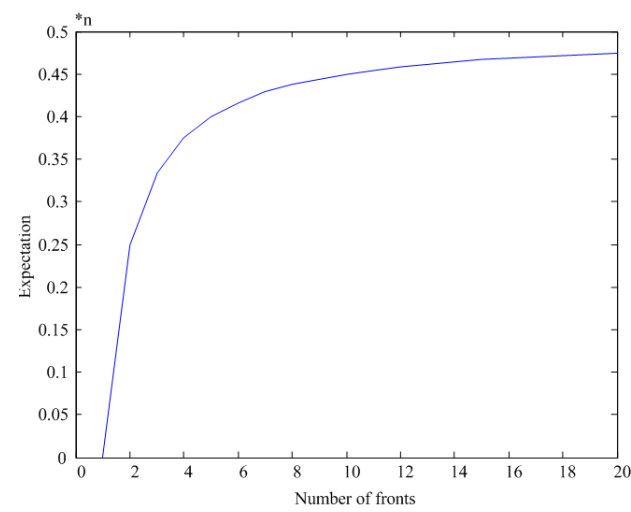

Fig. 9. The expected number of solutions that can be dominated with variable numbers of fronts in fixed front dataset.

ignores no solutions. Non-dominated rank sort just ignores a part of comparisons of the dominated solutions.

Figure 11 shows the result of average execution time on the fixed front dataset with variable numbers of fronts from 30 independent runs. Generally, the number of fronts does not influence execution time very much. Similar to the result on the cloud dataset, fast non-dominated sort costs the most execution time, deductive sort costs the second most execution time, and corner sort costs the least execution time among the four sorts.

Figure 12 is the result of numbers of comparisons on the fixed front dataset with variable numbers of objectives. From the result, we find that only the number of comparisons of fast non-dominated sort increases with the increasing number of objectives. Among these 4 kinds of sorts, corner sort requires the fewest comparisons.

Figure 13 is the result of average execution time on the fixed front dataset with variable numbers of objectives from 30 independent runs. The execution time of 4 non-dominated sorts is hardly influenced by the number of objectives. Corner sort costs the least time in most cases of the experiment.

\section{Experiment on Mix Dataset}

1) Mix Dataset: The last two kinds of datasets are uniformly distributed. Cloud dataset is distributed in the objective space. Fixed front dataset is distributed on fronts. In practical process of MOEAs, solutions are not distributed such uniformly. In view of this, we add an experiment on nonuniform datasets, i.e., mix dataset to test whether the distribution influences the performance of corner sort. Mix dataset is built by adding small size of cloud dataset to fixed front dataset with one front as Figure 14.

2) Results: The left subfigure of Figure 15 is the result of comparisons on the mix dataset with variable numbers of objectives. Fast non-dominated sort and non-dominated ranking sort require the first and second most comparisons respectively. For the mix dataset with low-dimensional objectives, corner sort requires more comparisons than deductive sort but the mix dataset with high-dimensional objectives, corner sort requires fewer comparisons than deductive sort, because it can save more objective comparisons in the process of obtaining nondominated solutions than deductive sort. The right subfigure of Figure 15 shows the result of execution time on the mix dataset with variable numbers of objectives. Fast non-dominated sort costs the most time. Non-dominated rank sort and corner sort cost the least time. In summary, uniformity does not influence non-dominated sorts.

\section{Experiment on Data generated from MOEAs}

1) Data generated from MOEAs: All the above experiments are applied on the artificial datasets, which cannot reflect the performance of non-dominated sorts in MOEAs. We adopted DTLZ1 $\left(\left|x_{g}\right|=5\right)$ and DTLZ2 $\left(\left|x_{g}\right|=10\right)$ with 2-30 objectives [32] for the experiment in this subsection. The data is from the populations of every generation in MOEAs. We run 200 generations on those benchmark problems with a population with 200 individuals in MOEAs. We adopt NSGA-II as the MOEA in the experiment. The parameter settings are shown in Table IV.

2) Results: There are 200 datasets for each problems. We conclude the results in Figure 16 with a error bar plot, which describes statistical information. In Figure 16, the numbers of comparisons of all the sorts except deductive sort have no influence with the number of objectives. Moreover, the execute time increases a little bit with the number of objectives. For DTLZ1, fast non-dominated sort requires both the most comparisons and the longest time. Corner sort requires both the fewest comparisons and the shortest time. The result of DTLZ2 is similar to that of DTLZ1.

Furthermore, the behaviors in different stages of MOEAs of different sorts are shown in Figure 17. In Figure 17, the number of comparisons is shown with different generations. Generally, corner sort performs best among these 4 sorts in all the stages. For 2-objective problems, non-dominated rank sort has worse performance at the beginning than that in other stages. Corner sort and deductive sort has better performance at the beginning than that in other stages. For MOPs with high dimensional objectives, the stages have little influence on all the sorts.

\section{E. Discussion and Analysis}

From these experiments above, we find corner sort save more execution time and comparisons than other comparative sorts on many-objective optimization problems. The advantage of corner sort is in the process of obtaining non-dominated solutions of many-objective problems. It only takes $N-1$ objective comparisons, which is fewer than other sorts. In the beginning of MOEAs, which is simulated by cloud dataset, corner sort outperforms others on many-objective problems. In the end of MOEAs, which is simulated by fix front dataset, corner sort still saves the most time and comparisons. The distribution of dataset has no influence on the efficiency of corner sort, which is shown by the experiment on mixed dataset. From these experiments on artificial datasets, we conclude those above. The experiment on the solution set generated from MOEAs shows that corner sort saves the most computational cost in whole procession of MOEAs. 

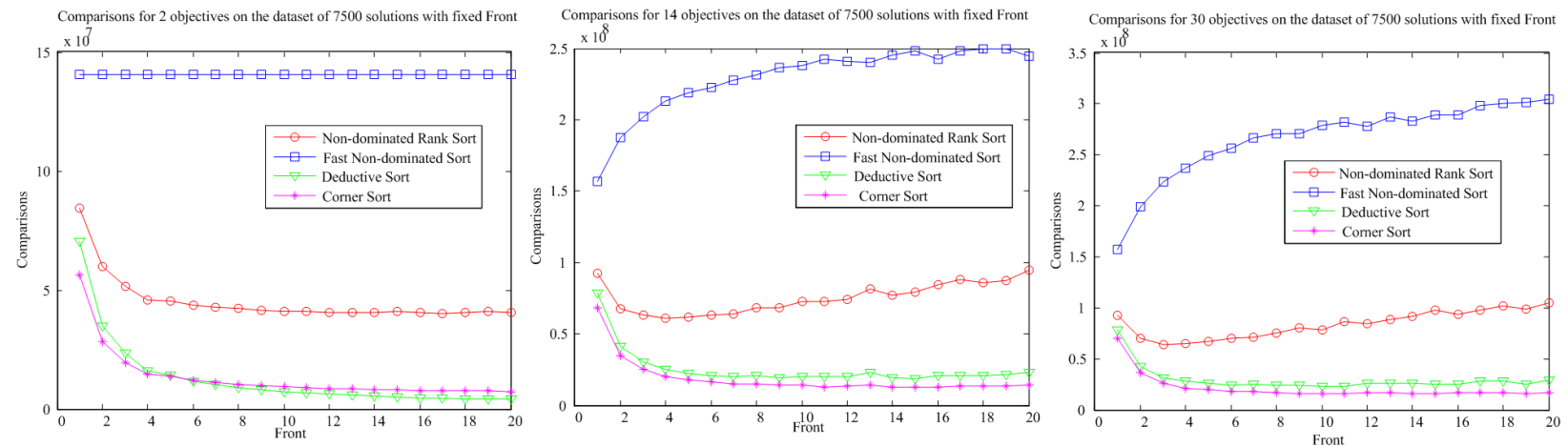

Fig. 10. Comparisons of 4 non-dominated sort on the fixed front datasets with 1 20 fronts of 7500 solutions.
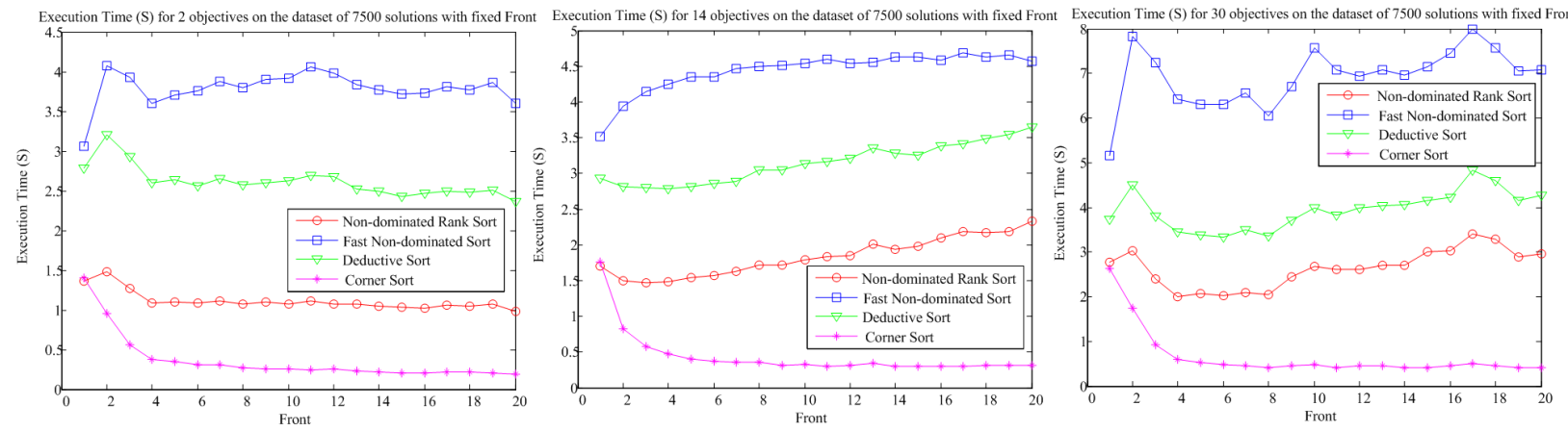

Fig. 11. Average execution time of 4 non-dominated sort on the fixed front datasets with 1 20 fronts of 7500 solutions from 30 independent runs.
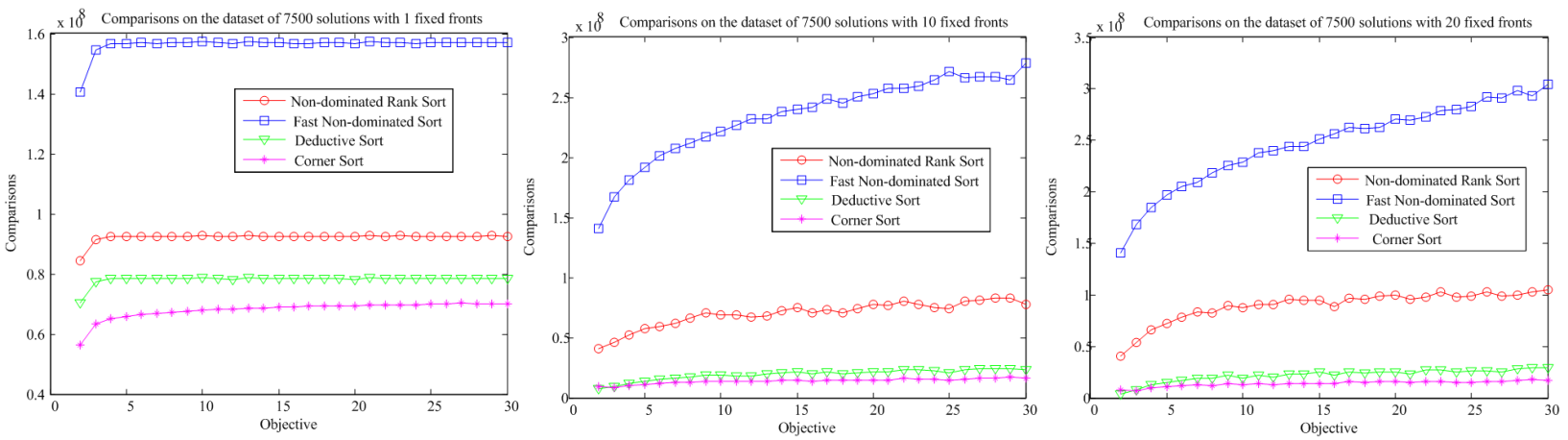

Fig. 12. Comparisons of 4 non-dominated sort on 1, 10, and 20 fixed front datasets with 2-30 objectives of 7500 solutions.
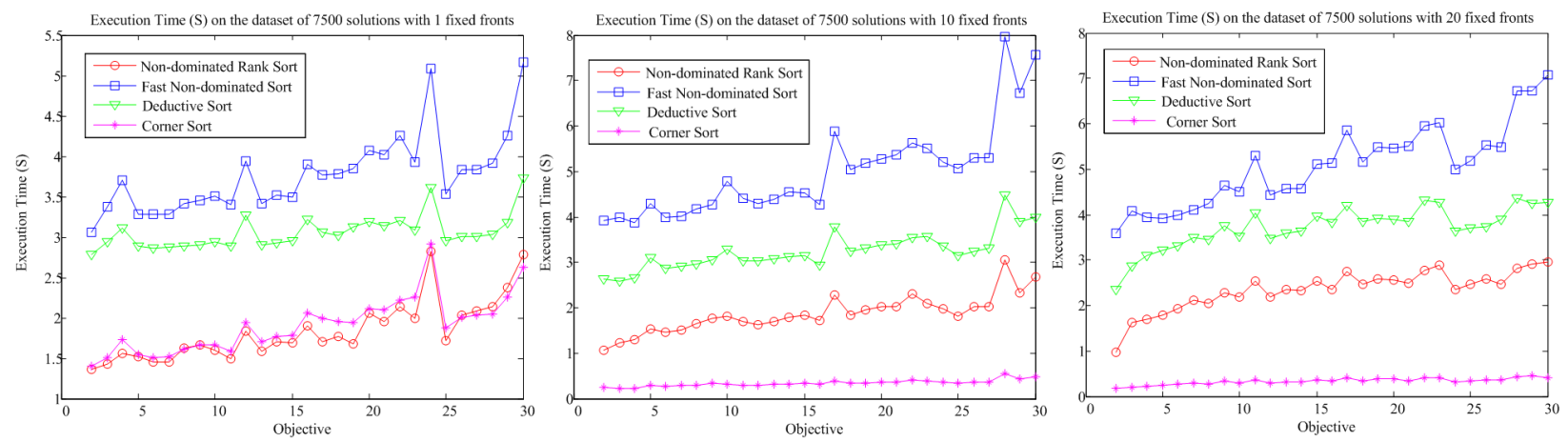

Fig. 13. Average execution time of 4 non-dominated sort on 1, 10, and 20 fixed front datasets from 30 independent runs. 
TABLE IV

PARAMETER SETTINGS FOR NSGA-II.

\begin{tabular}{cccc}
\hline Crossover & Mutation & Crossover ratio & Mutation ratio \\
\hline SBX & Polynomial mutation & 1 & $1 /($ dimension of decision variables $)$ \\
\hline
\end{tabular}

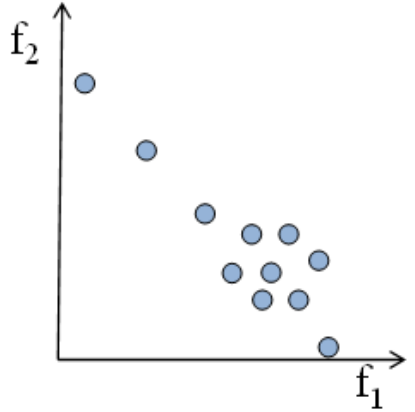

Fig. 14. An example of mix front dataset. Mix front is structured by a fixed front dataset (one front) and a cloud dataset of smaller size.

Although the experiment on the benchmark problem reflects the behaviors of different sorts, the problems in the real world are different from the benchmark. However, we still can infer that un-normalized objectives would not influence the behavior of corner sort because of its separate sorting on objectives.

Theoretically, all those four comparative sorts have the same level of computational complexity in the worst case. In the worst case, fast dominated sort need $m N(N-1)$ objective comparisons, while others need $m N(N-1) / 2$ objective comparisons. Obviously, fast dominated sort is the worst one without any comparison saving strategies. Thus, the analysis for other sorts is necessary. Although they require the same number of objective comparisons in the worst case, they have different comparison saving methods. Figure 18 shows their differences. All those sorts use a candidate solution to ignore the comparisons of the dominated solutions. However, the saved comparisons come from different ways. Non-dominated rank sort only saves the comparisons to the rest solutions when the candidate solution is dominated by any solutions. Deductive sort also saves those comparisons. At the same time, it saves the comparisons of the solutions which are dominated by the candidate solution. Deductive sort saves more comparisons than non-dominated sort definitely. Corner sort obtains a non-dominated solution by only $N-1$ objective comparisons, which saves comparisons. As the candidate solution is non-dominated, the first part of saved comparisons in Figure 18 cannot be obtained by corner sort. By the nondominated candidate solution, corner sort saves the second part of comparisons in Figure 18 as deductive sort. It seems to be hard to compare deductive sort and corner sort, which depends on the situation of the solution set. Namely, when the third part saves more comparisons than the first one, corner sort is better than deductive sort. As the analysis of Section III-C shows, the more objectives an MOP has, the more objective comparisons corner sort saves by obtaining non-dominated solutions. Moreover, there is less chance of a solution to be dominated in the solution set of many-objective problem. Few comparisons can be saved by the first part when faced many-objective problems. Therefore, corner sort saves more comparisons than deductive sort. That is the reason why corner sort performs best among the comparative sorts on many-objective optimization problems.

\section{CONClusion}

A novel corner sort for Pareto-based many-objective optimization is proposed in this paper. The proposed sort aims at saving comparison when obtaining non-dominated solutions. It saves more objective comparisons and execution time than other sorts on many-objective optimization problems according to our comparative experiments.

Although corner sort performs well in many-objective problems, its performances on MOPs with low-dimensional objectives are not satisfactory. We hope to improve corner sort further in our future work. As the proposed sort can obtain $m$ non-dominated solutions at the same time, we hope to develop the current sort into a parallel version. Moreover, the order of choosing non-dominated solution in corner sort affects its behavior according to different cases of solution set. In the future, we hope to improve it by adding a self-adaptive one.

\section{ACKNOWLEDGMENT}

This work was supported by an EU FP7 IRSES grant (no. 247619) on "Nature Inspired Computation and its Applications (NICaiA)", the National Natural Science Foundation of China, under Grants 61001202, and the National Research Foundation for the Doctoral Program of Higher Education of China, under Grants 20100203120008. Xin Yao was supported by a Royal Society Wolfson Research Merit Award.

\section{REFERENCES}

[1] C. Fonseca, P. Fleming et al., "Genetic algorithms for multiobjective optimization: Formulation, discussion and generalization," in Proceedings of the 5th international Conference on Genetic Algorithms, vol. 1, 1993, pp. 416-423.

[2] N. Srinivas and K. Deb, "Muiltiobjective optimization using nondominated sorting in genetic algorithms," Evolutionary computation, vol. 2, no. 3, pp. 221-248, 1994.

[3] K. Deb, A. Pratap, S. Agarwal, and T. Meyarivan, "A fast and elitist multiobjective genetic algorithm: NSGA-II," Evolutionary Computation, IEEE Transactions on, vol. 6, no. 2, pp. 182-197, 2002.

[4] J. Horn, N. Nafpliotis, and D. Goldberg, "A niched pareto genetic algorithm for multiobjective optimization," in Evolutionary Computation, 1994. CEC 1994. IEEE Congress on. IEEE, 1994, pp. 82-87.

[5] E. Zitzler and L. Thiele, "Multiobjective evolutionary algorithms: A comparative case study and the strength pareto approach," Evolutionary Computation, IEEE Transactions on, vol. 3, no. 4, pp. 257-271, 1999.

[6] E. Zitzler, M. Laumanns, L. Thiele et al., "SPEA2: Improving the strength pareto evolutionary algorithm," in Proceedings of EUROGEN 2001. Evolutionary Methods for Design, Optimization and Control With Applications to Industrial Problems. Citeseer, 2001, pp. 1-21.

[7] J. Knowles and D. Corne, "The pareto archived evolution strategy: A new baseline algorithm for pareto multiobjective optimisation," in Evolutionary Computation, 1999. CEC 1999. IEEE Congress on, vol. 1. IEEE, 1999, pp. 98-105. 

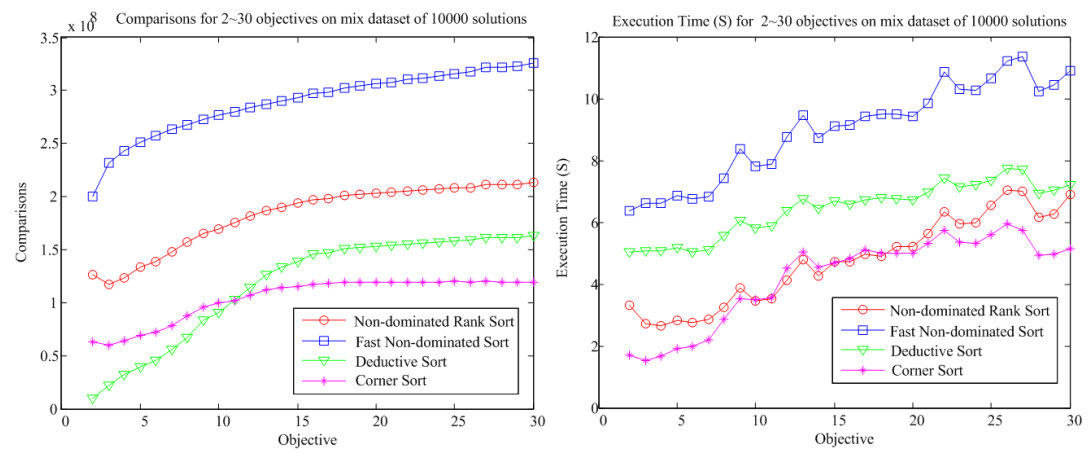

Fig. 15. Results of 4 non-dominated sort on mix datasets with $2-30$ objectives.
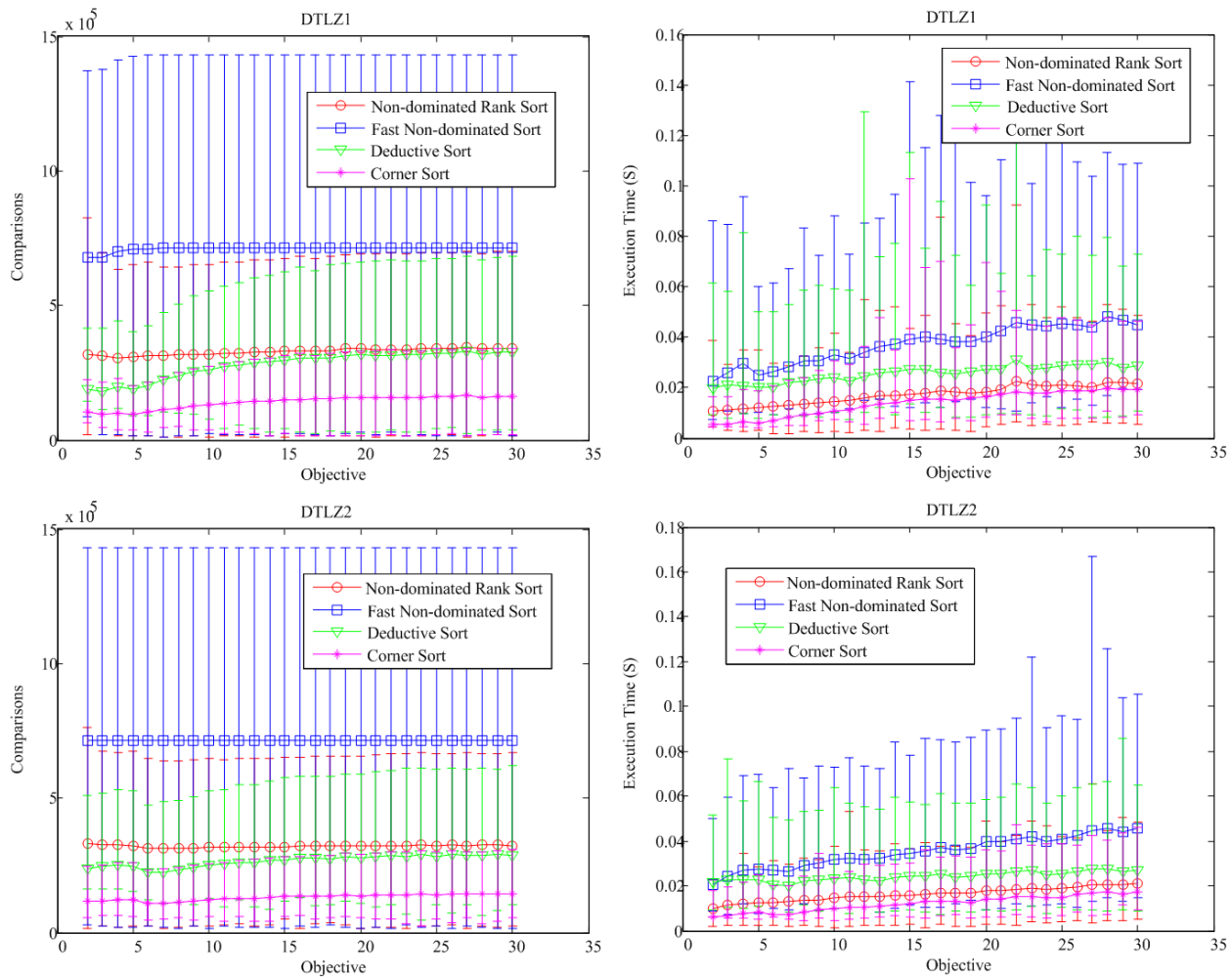

Fig. 16. Results of 4 non-dominated sort on the datasets from MOEAs with 2-30 objectives.

[8] D. Corne, N. Jerram, J. Knowles, M. Oates et al., "PESA-II: Regionbased selection in evolutionary multiobjective optimization," in Proceedings of the Genetic and Evolutionary Computation Conference (GECCO'2001), 2001, pp. 283-290.

[9] C. Coello Coello and M. Lechuga, "MOPSO: A proposal for multiple objective particle swarm optimization," in Evolutionary Computation, 2002. CEC 2002. IEEE Congress on, vol. 2. IEEE, 2002, pp. 10511056.

[10] İ. Karahan and M. Koksalan, "A territory defining multiobjective evolutionary algorithms and preference incorporation," Evolutionary Computation, IEEE Transactions on, vol. 14, no. 4, pp. 636-664, 2010.

[11] V. Khare, X. Yao, and K. Deb, "Performance scaling of multi-objective evolutionary algorithms," in Evolutionary Multi-Criterion Optimization, ser. Lecture Notes in Computer Science, C. Fonseca, P. Fleming, E. Zitzler, L. Thiele, and K. Deb, Eds. Springer Berlin / Heidelberg, 2003, vol. 2632, pp. 376-390.

[12] K. Praditwong and X. Yao, "How well do multi-objective evolutionary algorithms scale to large problems," in Evolutionary Computation, 2007. CEC 2007. IEEE Congress on. IEEE, 2007, pp. 3959-3966.

[13] T. Wagner, N. Beume, and B. Naujoks, "Pareto-, aggregation-, and indicator-based methods in many-objective optimization," in Evolution- ary Multi-Criterion Optimization. Springer, 2007, pp. 742-756.

[14] H. Ishibuchi, N. Tsukamoto, and Y. Nojima, "Evolutionary manyobjective optimization: A short review," in Evolutionary Computation, 2008. CEC 2008. IEEE Congress on. IEEE, 2008, pp. 2419-2426.

[15] O. Schutze, A. Lara, and C. Coello, "On the influence of the number of objectives on the hardness of a multiobjective optimization problem," Evolutionary Computation, IEEE Transactions on, vol. 15, no. 4, pp. 444-455, 2011.

[16] D. Brockhoff and E. Zitzler, "Improving hypervolume-based multiobjective evolutionary algorithms by using objective reduction methods," in Evolutionary Computation, 2007. CEC 2007. IEEE Congress on. IEEE, 2007, pp. 2086-2093.

[17] A. López Jaimes, C. Coello Coello, and D. Chakraborty, "Objective reduction using a feature selection technique," in Proceedings of the Genetic and Evolutionary Computation Conference (GECCO'2008). ACM, 2008, pp. 673-680.

[18] H. Ishibuchi, Y. Sakane, N. Tsukamoto, and Y. Nojima, "Evolutionary many-objective optimization by NSGA-II and MOEA/D with large populations," in Systems, Man and Cybernetics, 2009. SMC 2009. IEEE International Conference on. IEEE, 2009, pp. 1758-1763.

[19] E. Zitzler and S. Künzli, "Indicator-based selection in multiobjective 

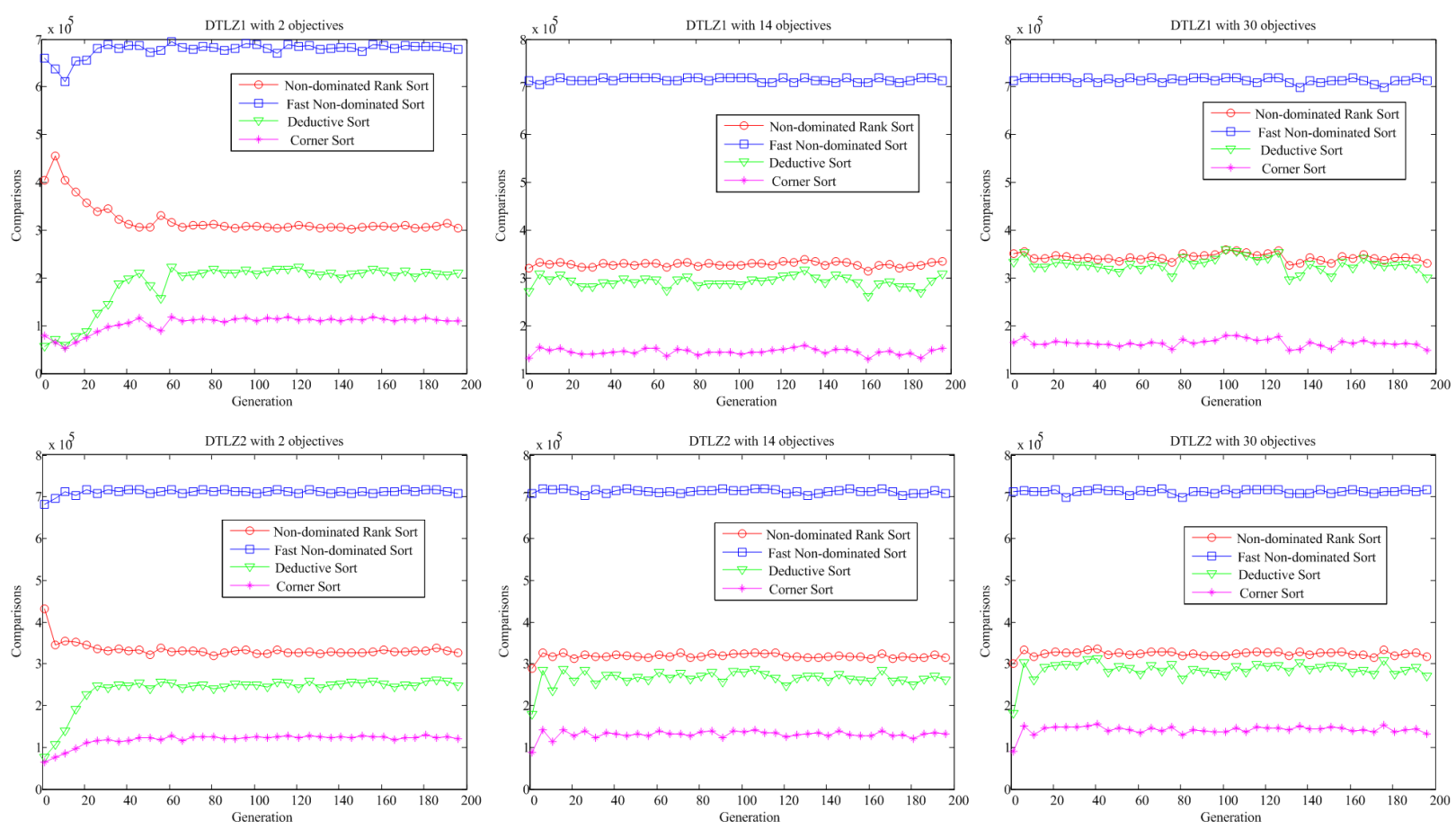

Fig. 17. Comparisons of 4 non-dominated sort on 2, 14, 30 objectives using the datasets from MOEAs in different generations.

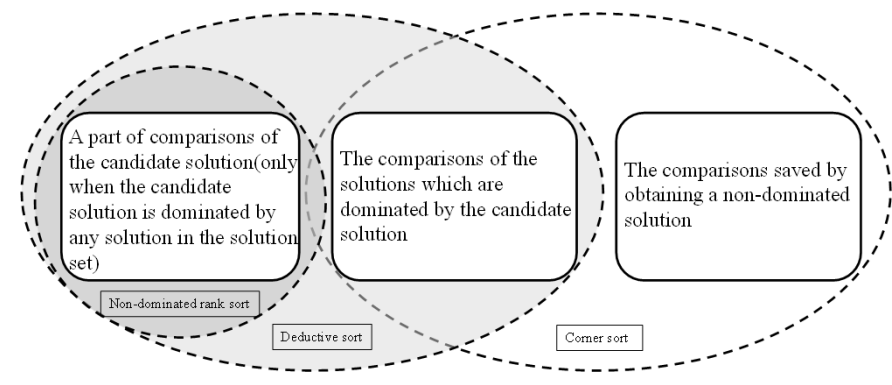

Fig. 18. The illustration of comparisons saved by different non-dominated sorts.

search," in Parallel Problem Solving from Nature-PPSN VIII. Springer, 2004, pp. 832-842.

[20] J. Bader and E. Zitzler, "A hypervolume-based optimizer for highdimensional objective spaces," in New Developments in Multiple Objective and Goal Programming, ser. Lecture Notes in Economics and Mathematical Systems, D. Jones, M. Tamiz, and J. Ries, Eds. Springer Berlin Heidelberg, 2010, vol. 638, pp. 35-54.

[21] E. Hughes, "Many-objective directed evolutionary line search," in Proceedings of the Genetic and Evolutionary Computation Conference (GECCO'2011). ACM, 2011, pp. 761-768.

[22] H. Sato, H. Aguirre, and K. Tanaka, "Controlling dominance area of solutions and its impact on the performance of MOEAs," in Evolutionary Multi-Criterion Optimization. Springer, 2007, pp. 5-20.

[23] H. Aguirre and K. Tanaka, "Space partitioning with adaptive $\varepsilon$-ranking and substitute distance assignments: a comparative study on manyobjective mnk-landscapes," in Proceedings of the Genetic and Evolutionary Computation Conference (GECCO'2009). ACM, 2009, pp. 547-554.

[24] M. Köppen, R. Vicente-Garcia, and B. Nickolay, "Fuzzy-paretodominance and its application in evolutionary multi-objective optimization," in Evolutionary Multi-Criterion Optimization. Springer, 2005, pp. $399-412$.

[25] M. Köppen and C. Veenhuis, "Multi-objective particle swarm optimization by fuzzy-pareto-dominance meta-heuristic," International Journal of Hybrid Intelligent Systems, vol. 3, no. 4, pp. 179-186, 2006.
[26] O. Schütze, "A new data structure for the nondominance problem in multi-objective optimization," in Evolutionary Multi-Criterion Optimization. Springer, 2003, pp. 509-518.

[27] M. Lukasiewycz, M. Glaß, C. Haubelt, and J. Teich, "Symbolic archive representation for a fast nondominance test," in Evolutionary MultiCriterion Optimization. Springer, 2007, pp. 111-125.

[28] H. Singh, A. Isaacs, and T. Ray, "A pareto corner search evolutionary algorithm and dimensionality reduction in many-objective optimization problems," Evolutionary Computation, IEEE Transactions on, vol. 15, no. 4 , pp. 539-556, 2011.

[29] M. Koppen, K. Yoshida, and K. Ohnishi, "A GRATIS theorem for relational optimization," in Hybrid Intelligent Systems (HIS), 2011 11th International Conference on. IEEE, 2011, pp. 674-679.

[30] K. Deb and S. Tiwari, "Omni-optimizer: A procedure for single and multi-objective optimization," in Evolutionary Multi-Criterion Optimization. Springer, 2005, pp. 47-61.

[31] K. McClymont and E. Keedwell, "Deductive sort and climbing sort: New methods for non-dominated sorting," Evolutionary Computation, vol. 20, no. 1, pp. 1-26, 2012.

[32] K. Deb, L. Thiele, M. Laumanns, and E. Zitzler, "Scalable multiobjective optimization test problems," in Evolutionary Computation, 2002. CEC 2002. IEEE Congress on. IEEE), 2002, pp. 825-830. 